\title{
Vasculitis crioglobulinémica con compromiso renal y síndrome de Sjögren
}

\section{Cryoglobulinemic vasculitis with renal involvement and Sjögren's syndrome}

\author{
María Emilia Debernardi ${ }^{1}$, Candela Preti ${ }^{2}$, Cindy Paula Frare ${ }^{2}$, Gabriela Bendjuia ${ }^{3}$ y María C. Corbella ${ }^{4}$
}

\section{RESUMEN}

La vasculitis crioglobulinémica es una vasculitis de vasos pequeños que se da en presencia de crioglobulinas séricas. Las crioglobulinemias mixtas son las más frecuentes y se asocian a infecciones crónicas, típicamente al virus de la hepatitis $C$, así como a enfermedades linfoproliferativas y autoinmunes, más a menudo al síndrome de Sjögren. Las manifestaciones clínicas incluyen púrpura de los miembros inferiores, neuropatía periférica, artralgias y glomerulonefritis. La presencia de vasculitis crioglobulinémica en el contexto de un síndrome de Sjögren es marcadora de peor pronóstico.
Presentamos el caso de una paciente con vasculitis crioglobulinémica con compromiso cutáneo y renal, que condujo al diagnóstico de un síndrome de Sjögren primario.

Palabras clave: síndrome de Sjögren, vasculitis leucocitoclástica, vasculitis crioglobulinémica, crioglobulinemia, púrpura de los miembros inferiores, glomerulonefritis.

Dermatol. Argent. 2020, 26 (1): 42-44

\section{ABSTRACT}

Cryoglobulinemic vasculitis is a small-sized vasculitis that occurs in the context of serum cryoglobulins. Mixed cryoglobulinemias are the most frequent and are associated with chronic infections, typically hepatitis C and autoimmune diseases, most commonly Sjögren's syndrome. Clinical manifestations include purpura of lower limbs, peripheral neuropathy, arthralgias and glomerulonephritis. The presence of cryoglobulinemic vasculitis in the context of Sjögren's syndrome is a marker of poor prognosis. We present the case of a patient with cryoglobulinemic vasculitis associated to cutaneous and renal involvement that led us to the diagnosis of primary Sjögren's syndrome.

Key words: Sjögren's syndrome, leukocytodlastic vasculitis, cryoglobulinemic vasculitis, cryoglobulinemia, purpura of lower limbs, glomerulonephritis.

Dermatol. Argent. 2020, 26 (1): 42-44
1 Médica de la Carrera de Especialista en Dermatología, Universidad de Buenos Aires. Especialista en Medicina Interna

2 Médica Especialista en Dermatología

${ }^{3}$ Médica Especialista en Dermatología y Medicina Interna, Médica de Planta, Sector Colagenopatías

${ }^{4}$ Médica Especialista en Anatomía Patológica

División Dermatología, Hospital General de Agudos Dr. J. M.

Ramos Mejía, Ciudad Autónoma de Buenos Aires, Argentina
Contacto del autor: María Emilia Debernardi

E-mail: emiliadebernardi@gmail.com

Fecha de trabajo recibido: 10/1/2020

Fecha de trabajo aceptado: 12/3/2020

Conflicto de interés: las autoras declaran que no existe conflicto de interés.

\section{CASO CLÍNICO}

Una mujer de 67 años consultó por una dermatosis localizada en los miembros inferiores que aparecía en el contexto de la bipedestación prolongada y se resolvía con el reposo, de 3 ańos de evolución. Las lesiones eran dolorosas y levemente pruriginosas. Como antecedentes personales presentaba cavernomatosis portal secundaria a déficit de proteína $\mathrm{C}$, hipertensión portal con hiperesplenismo y várices esofagogástricas, trombosis venosa profunda y tromboflebitis de repetición, en tratamiento con propranolol y enoxaparina.

En el examen físico presentaba máculas violáceas redondeadas de contornos definidos, de aproximadamente $3 \mathrm{~cm}$ de diámetro, negativas a la vitropresión, localizadas en los miembros inferiores (Foto 1). 


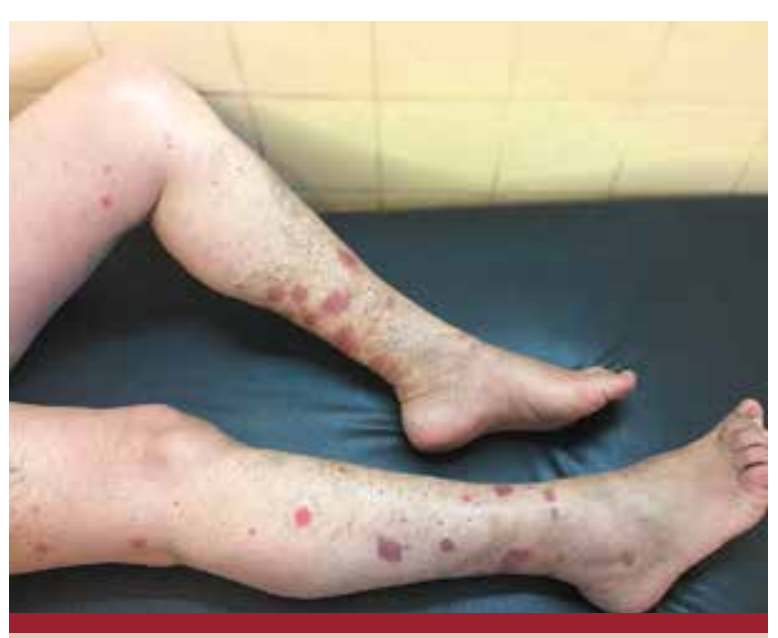

F0T0 1: Máculas violáceas redondeadas de contornos definidos de aproximadamente $3 \mathrm{~cm}$ de diámetro, vitropresión negativa, localizadas en los miembros inferiores.

El análisis de laboratorio evidenció una función renal normal; serologías para HIV, hepatitis B y C (HCV) negativas; PCR para HCV negativa; hipocomplementemia (C3 $61 \mathrm{mg} / \mathrm{dl}, \mathrm{C} 41 \mathrm{mg} / \mathrm{dl}, \mathrm{CH} 50<$ $10 \mathrm{mg} / \mathrm{d})$; factor reumatoideo positivo; ANA (1/160) con patrón moteado; anti-Ro positivo, anti-DNA, anti-Sm, anti-La, c-ANCA y p-ANCA negativos. La determinación de crioglobulinas fue positiva (en tres oportunidades) y el proteinograma electroforético mostró una hipergammaglobulinemia policlonal. El sedimento urinario presentó 15-20 glóbulos rojos por campo y la proteinuria de 24 horas fue de $1,3 \mathrm{~g}$. La biopsia de piel de la pierna izquierda informó vasculitis leucocitoclástica de pequeños vasos (Foto 2) con inmunofluorescencia directa (IFD) positiva en las paredes vasculares para IgG, C3 y fibrinógeno. La biopsia renal mostró glomerulonefritis membranoproliferativa de tipo I con $30 \%$ de esclerosis y $20 \%$ de obleas. Ante estos resultados, se interrogó nuevamente a la paciente, que refirió artralgias y xerostomía, por lo que se realizó una biopsia de la glándula salival menor, que informó sialadenitis linfocítica con un componente inflamatorio vinculable al síndrome de Sjögren (SS) (focus score: 1,69).

Con estos hallazgos se arribó al diagnóstico de crioglobulinemia tipo III y vasculitis crioglobulinémica (VC) con compromiso renal en el contexto de un síndrome de Sjögren primario (SSp). Se inició tratamiento con meprednisona $40 \mathrm{mg} /$ día y azatioprina $150 \mathrm{mg} /$ día, asociado a furosemida, irbesartán y enalapril por el compromiso renal. Desde el punto de vista cutáneo, la paciente evolucionó de manera favorable y continuó en seguimiento por nefrología, con descenso progresivo de la proteinuria y función renal estable.

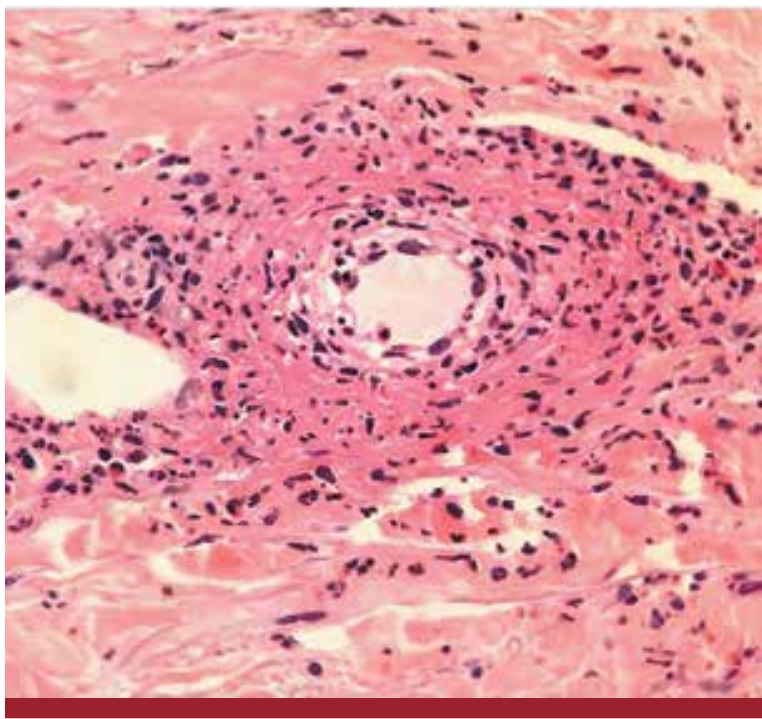

F0T0 2: Piel del miembro inferior derecho. Vaso de pequeño calibre con necrosis fibrinoide, infiltrado inflamatorio compuesto por polimorfonucleares en el espesor del vaso y alrededor de este, asociado a extravasación eritrocitaria (HyE, 400X).

\section{COMENTARIOS}

Las crioglobulinas son inmunoglobulinas presentes en el suero que precipitan in vitro a temperaturas inferiores a los $37^{\circ} \mathrm{C}$. Brouet et ál. clasificaron las crioglobulinemias según el tipo de inmunoglobulina presente en tipo I (IgM o IgG monoclonal), tipo II (IgM monoclonal asociada a IgG policlonal) y tipo III (IgG policlonal asociada a IgM policlonal) ${ }^{1,2}$.

En la de tipo I, las inmunoglobulinas se producen por algún trastorno linfoproliferativo clonal de células B subyacente y generan daño vascular por hiperviscosidad y trombosis. Las de tipos II y III se denominan mixtas (IgG más IgM) y son secundarias a infecciones, principalmente al HCV (60-90\%), y, en segundo lugar, a enfermedades autoinmunes, más a menudo al SS como fue el caso de esta paciente ${ }^{3}$. El mecanismo propuesto para el desarrollo de vasculitis es la precipitación de inmunoglobulinas y la formación de inmunocomplejos en los vasos de pequeño calibre de la piel, articulaciones, nervios periféricos y riñón ${ }^{4}$.

La tríada clínica característica de púrpura, artralgias y debilidad muscular suele estar presente desde el inicio, como sucedió en este caso, sin la debilidad muscular ${ }^{5}$. Otras manifestaciones incluyen neuropatía periférica $(60 \%)$ y nefropatía $(30 \%)^{1}$.

Entre el 5,3\% y el $28 \%$ de los pacientes con SS primario (SSp) presentan crioglobulinas positivas, pero solo un 3-4\% desarrollarán $\mathrm{VC}^{6,7}$. La presencia de VC se asocia a mayor compromiso extraglandular, aumento del riesgo de linfoma B y menor sobrevida ${ }^{8}$. 
La VC suele localizarse en los miembros inferiores como púrpura palpable y el hallazgo histológico más común es la vasculitis leucocitoclástica con IFD positiva para $\mathrm{IgG}$, $\mathrm{IgM}$ y $\mathrm{C} 3^{7}$.

El tratamiento de la VC dependerá de la etiopatogenia y la severidad del cuadro clínico. En los casos leves a moderados asociados a SSp, consiste en evitar las bajas temperaturas, reposo y fármacos como antiinflamatorios no esteroides, colchicina o dapsona.

\section{BIBLIOGRAFÍA}

1. Ramos-Casals $M$, Stone $J H$, Cid MC, Bosch $X$. The cryoglobulinaemias. Lancet 2012;379(9813):348-360.

2. Brouet JC, Clauvel JP, Danon F, Klein M, et ál. Biologic and clinical significance of cryoglobulins. A report of 86 cases. Am J Med 1974;57:775-788.

3. Cacoub P, Comarmond C, Domont F, Savey L, et ál. Cryoglobulinemia vasculitis. Am J Med 2015;128:950-955.

4. Terrier B, Cacoub P. Cryoglobulinemia vasculitis: an update. Curr Opin Rheumatol 2013;25:10-18.

5. Ferri C, Sebastiani M, Giuggioli D, Cazzato M, et ál. Mixed cryoglobulinemia: demographic, clinical, and serologic features and survival in 231 patients. Semin Arthritis Rheum 2004;33:355-374.

6. Baldini C, Pepe P, Quartuccio L, Priori R, et ál. Primary Sjogren's syndrome as a multi-organ disease: impact of the
En casos graves con compromiso sistémico, se utilizan corticosteroides; inmunosupresores como micofenolato, azatioprina y ciclofosfamida; rituximab, o plasmaféresis ${ }^{4,9}$.

Destacamos el rol del médico dermatólogo en el diagnóstico precoz de una enfermedad sistémica a través de sus manifestaciones cutáneas. Por otro lado, resaltamos el manejo multidisciplinario en el tratamiento y el seguimiento de estos pacientes.

serological profile on the clinical presentation of the disease in a large cohort of Italian patients. Rheumatology (Oxford) 2014;53:839-844.

7. Singh AG, Singh S, Matteson EL. Rate, risk factors and causes of mortality in patients with Sjögren's syndrome: a systematic review and meta-analysis of cohort studies. Rheumatology (Oxford) 2016;55:450-460.

8. Retamozo S, Gheitasi L, Quartuccio L, Kostov B, et ál. Cryoglobulinaemic vasculitis at diagnosis predicts mortality in primary Sjögren syndrome: analysis of 515 patients. Rheumatology 2016;55:1443-1451.

9. Silva F, Pinto C, Barbosa A, Borges T, et ál. New insights in cryoglobulinemic vasculitis. J Autoimmun 2019;105:102313.

\section{DERMATÓLOGOS JÓVENES}

\section{^ Elección múltiple: LESIONES PURPÚRICAS EN LOS MIEMBROS INFERIORES}

\section{Paula Johana Barba, Pilar Targize Vaquero y Florencia Guillermina Martínez \\ Servicio de Dermatología, HIGA Prof. Dr. Rodolfo Rossi, La Plata, Provincia de Buenos Aires, Argentina}

Una mujer de 62 años, con antecedentes de hipotiroidismo, dislipidemia e hipertensión arterial en tratamiento, consultó por la presencia de una dermatosis pruriginosa en ambas piernas, de 7 meses de evolución. Examen físico dermatológico: presentaba en las caras anterior y lateral de los miembros inferiores placas eritemato-anaranjadas, algunas de límites netos, otras de límites difusos, con petequias en su superficie (Fotos 1, 2 y 3).

\section{1) ¿Cuál es su diagnóstico más probable?}

A. Púrpura por alteraciones hematológicas. B. Vasculitis cutánea de vasos pequeños. C. Dermatosis purpúrica pigmentaria. D. Dermatitis de contacto alérgica. E. Dermatitis por estasis.

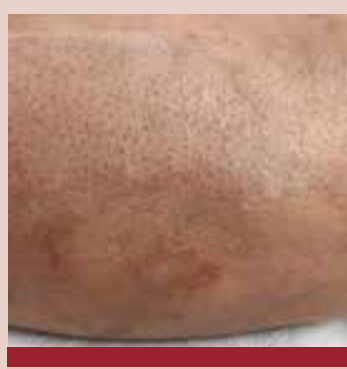

FOTO 1: Placas eritemato-anaranjadas en la pierna izquierda.

2) Los hallazgos que descartan esta entidad son:

A. Inflamación de células endoteliales con extravasación de glóbulos rojosy macrófagos que contienen hemosiderina. B. Alteración del tiempo de sangría o del coagulograma.

C. Vasculitis leucocitoclástica.

D. Eco-Doppler venoso de los miembros inferiores alterado. E. Las opciones B. y C. son correctas.

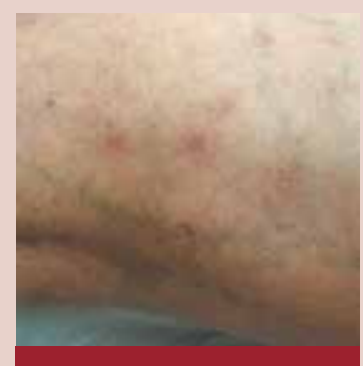

F0T02: Lesiones petequiales puntiformes.

3) ¿Qué enunciado considera verdadero basado en el cuadro de sospecha?

A. La mayoría son crónicas y no asocian compromiso sistémico. B. El compromiso cutáneo puede ser único o estar asociado a compromiso de órganos internos.

C. Son más frecuentes en los miembros inferiores y pueden producir prurito de intensidad variable.

D. Las respuestas A. y C. son correctas. E. Las respuestas B. yC. son correctas.

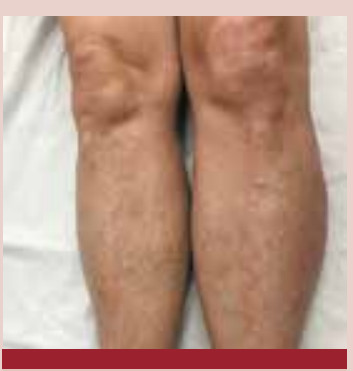

FOTO 3: Lesiones localizadas en la cara anterior de ambas piernas.

4) ¿Qué tratamiento indicaría en esta paciente según su diagnóstico presuntivo?

A. Transfusión de hemoderivados. B. Corticosteroides tópicos + antihistamínicos orales.

C. Corticosteroides sistémicos. D. Corticosteroides sistémicos + inmunosupresores.

E. Todas las opciones son correctas. La solución en la página 48 